\title{
Design Innovation for Intergenerational Services
}

\author{
Sara Ines Sanchez Alquijay ${ }^{1}$ \\ Department of Interaction Design, National Taipei University of Technology \\ No. 1, Section 3, Zhongxiao East Road, Da'an District, Taipei, Taiwan \\ E-mail:t106ac8401@ntut.edu.tw

\section{Camilo Jaime Gómez} \\ Department of Interaction Design, National Taipei University of Technology \\ No. 1, Section 3, Zhongxiao East Road, Da'an District, Taipei, Taiwan \\ E-mail:t107at8409@ntut.edu.tw
}

\section{Ryan Sheng-Ming Wang, Ph. D.}

Department of Interaction Design, National Taipei University of Technology

No. 1, Section 3, Zhongxiao East Road, Da'an District, Taipei, Taiwan

E-mail: ryan5885@mail.ntut.edu.tw

Integration between generations is an opportunity for increased mutual understanding that results from the interaction between both elder and youth, and that benefits independently and as a society level. While it is not always possible for generations to be in close proximity very often, it is important for both youth and seniors to connect with those of different generations. Intergenerational relationships can help people feel a greater sense of fulfilment and with the rapid technological development, innovative services offer a promising platform to achieve this. Design practices and technology offer now an interdisciplinary integrated approach to assess services development and evaluate service innovation. Based on an interdisciplinary integration framework and qualitative research, this study combines the usage of service design tools to develop and prototype an intergenerational platform that enables the connection of elder adults and youth through task service and collaboration. A structural and an empirical service design analysis was developed using an integration of design thinking analysis and service design assessment, and the system was evaluated through a Kano model analysis to support the design innovation creation of a service platform that connects generations through technology.

\section{${ }^{1}$ Sara Ines Sanchez Alquijay}


International Symposium on Grids \& Clouds 2019, ISGC2019

31st March - 5th April, 2019

Academia Sinica, Taipei, Taiwan 
1. Introduction

When older adults contribute to the well-being of youth, it cultivates a sense of purpose and extends the benefits both ways. Interactions with young people allow older adults to relate to another generation and learn about new technologies and trends. It also let's older people to experience the excitement of seeing the world through a younger perspective. This can get older adults up and motivated, reducing depression, relieving boredom and improving health. Young people benefit too. The attention and example provided by a senior can be invaluable. Elder serve as role models for children growing and such relationships are important for society. They can help ensure that youngsters receive the kind of attention and guidance they might lack.

Several reasons can help us understand why integrating both generations can be beneficial. First, changes in both youth and old age have created an opportunity for elder people to meet the needs of young people. In general, youth depends upon adults for care, training, supervision, and transfer of material resources.

A second reason for integrating older and younger is the potential for increased mutual understanding that results from the interaction between both. Stereotypes from age are nurtured in an environment of high segregation. Development of cross-age relationships could decrease and change biases that old people have toward children and adolescents and vice versa.

A third reason and a very relevant one for this study involves the opportunity for youngsters to meet the needs of elder people. Youth needs to be involved in meeting the needs of the growing number of elders in the population. And there are many special opportunities for young people to be involved in this task. Young people have skills and energy that could be channel towards helping elder people.

From understanding this, we propose an intergenerational platform that enabled by technology connects elder and youngsters through task service and collaboration. This was done with the purpose of applying innovative management tools for service development validation, and to contribute in the aspect of doing social innovation design for platforms that promote interaction between generations.

The objective of this study is to provide a detail explanation of our innovative proposition and service development process that can be used for product or service development or even as an innovative development tool by organizations when starting from zero or innovating an existing service at an internal level.

\section{Literature Review}

\subsection{Related Literature}

The intergenerational gap which causes loneliness among seniors and makes them depended on the help of the family or nursing homes has been seen as an increasing problem nowadays. Extended families are decreasing and therefore youngsters have little contact with elder ones. Seniors - living alone or in institutions - disappear from public life and become one marginal group [1]. Youth then have no way of meeting elder adults nor building a relationship with them. As a result, both sides lack the opportunity to connect.

The aging population has grown rapidly in Taiwan. As foreseen by the EDP (National Economic Development Program), by 2017, the aging population in Taiwan exceeded $14 \%$ of the total population and the country officially became an "old society" [2]. Changes in the structure 
of the population and the composition of the family affect the economic and social situation not only in Taiwan but in the world as well.

On the other hand, Service Design tools, the Design Thinking methodology, the Business Model Canvas and the Kano Model are innovative tools for product and service development. These tools are used either to validate a new project or an existing one and they offer an innovative holistic framework to fast develop a product or a service focusing in a co-creation process that brings everyone that is useful to the best output together as much as you can.

\subsection{Related Case Studies}

In recent years, different social businesses and non-profit projects evolved with focus on supporting and changing the traditional way to improve the mental health of older generations.

The idea of aging at home has been promoted through different projects focused in homecare improvement but also several volunteer organizations have been trying to close this gap with kindergartens connected to nursing homes as an approach to solve the growing phenomenon of the elderly. In the years 2000 and 2003, a nursing home in cooperation with an organization that runs several kindergartens in Vienna started a special project, a kindergarten integrated into a retirement home [3]. A meeting framework for old and young was created, setting an example for the present and future of many old and young generations. The integrated space allowed the encounter between elder and young, which often no longer exists in families, and it also promoted life skills development for both children and seniors.

Another example is myBestHelper which is a digital tool that offers families the easiest way to book helpers for children care based on their needs [4]. Their services help families find childcare or home care. Non-professional caregivers are available on the platform, on the assumption that a trustworthy person can be trained to do the job. The so-called helpers include a mix of university students, stay-at-home mothers and retirees looking for additional income.

\subsection{Problem}

Nowadays what used to be covered by the extended family has to be provided mostly by society. This means that institutions and organizations have to focus in providing services to create the appropriate framework and conditions so that dignified aging is possible. Specifically, in terms of housing, economy situation and the health of the elderly.

Some elder people are stable and active in their own and can manage their lives independently. Often, however, assistance is needed, be it in daily activities, grocery shopping, among others. For many seniors there is also a need for care. Even though there are alternatives, these are limited, and many older people have to live alone facing solitude and the challenge of accomplishing daily shores which might be hard for them due to their physical condition.

\section{Research Process}

\subsection{Design Thinking Analysis}

Dam \& Siang describe Design Thinking as an innovative design methodology that provides a solution-based approach to solving problems [5]. It's useful in tackling complex problems, by understanding the human needs involved, by re-framing the problem in human-centric ways, by creating ideas in brainstorming sessions, and by adopting an innovative hands-on approach in 
prototyping and testing. In this research we will focus on the five-stage Design Thinking model proposed by the Hasso-Plattner Institute of Design at Stanford University (d.school). The five stages of Design Thinking, according to the d.school, are as follows: Empathy, Define (the problem), Ideate, Prototype, and Test. For this research, a design thinking approach was used to evaluate the problem and understand how it could be validated. Fig. 1 refers to the flow followed through this research and it shows how the methodology was applied in the context of the information collected below and the identified problem.

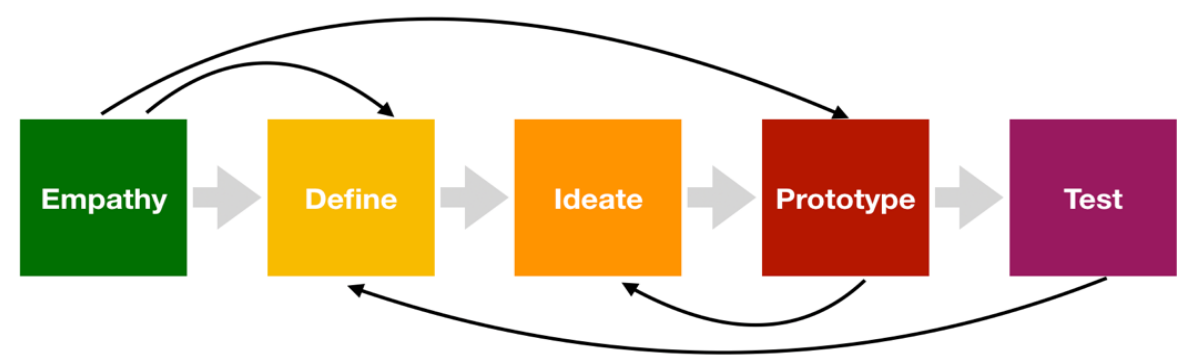

Fig. 1. Five stages of Design Thinking proposed by the Hasso-Plattner Institute of Design at Stanford University [5].

\subsubsection{Empathy}

This phase focuses in gaining an empathic understanding of the people designing for and the problem trying to be solved [5]. To gain empathic understanding on our future users, we observed and ran surveys to a group of elder people and youth. We learned that elder population is increasing in Taiwan and the solutions for the challenges they encounter in the everyday lives are limited. On the other hand, we learned that resources for students can be limited since during that time youth is focused in developing their skills and have limited time to work. As we mentioned before this information was collected through observations and surveys to understand the potential users (elders and youth). We had a total of 20 participants for each group.

Some of the insights collected from young people:

- "Would definitely do some "good favors" in exchange of coupons"

- "Of course, there is always that old woman or men that you want to help"

- "I would be interested in learning from someone with years of experience"

Some of the insights collected from elder people:

- "I really could use some help sometimes but I'm afraid to ask for it"

- "I'm afraid to interrupt my children while they're working or in their activities."

- "I just need someone to help with my groceries"

\subsubsection{Define}

During this phase, we put together the information collected and gathered during the empathy phase. Information and observations had to be analyzed and synthesized in order to define the core problems identified up to this point. Based on the observations and information collected:

a) Elder people need help and often lack of ways to reach for it or where to find it.

b) Elder people struggle with complex technology but are familiarized in using smartphones and tablets in their everyday. 
c) Many youngsters are open to opportunities that increase their resources or provide them with benefits.

\subsubsection{Ideate}

With a solid background, we started to identify solutions to the problem statements and look for different ways of viewing the problem. We ideated different ideas and chose the one that was aligned with the insights we had collected before. The idea was a platform that supports elder people that need help to complete daily life tasks - like grocery shopping or gardening - by connecting them with youngsters that apply to complete these tasks in exchange of some kind of reward. In plain speech, elderly people get the support and youngsters receive reward points in exchange for helping elderly people. These points can be later exchanged for discounts in clothe stores or restaurants.

\subsubsection{Prototype}

During this phase an inexpensive, scaled down version of the product or service was produced. The prototype was going to be useful to share and test the tool to get information from the solution created for the problem. We developed the prototype of our platform as a mobile app which we later called ShareCare.

\subsubsection{Test}

During this stage, we tested the mobile app to understand if we had built the solutions we had identified. This was not the final stage since as an innovative iterative process, the results generated during this phase where going to be used to redefine the problem and improve the user experience. Using the real prototype and surveys we were able to validate the prototype.

\subsection{Service Design Analysis}

Service design was first introduced as a design discipline at the Köln International School of Design in 1991. The purpose of service design methodologies is to design according to the needs of customers or participants, so that the service is user-friendly, competitive and relevant to the customers [6]. Many tools are involved in the process designing an innovative service. A service design analysis was done to plan and organize the resources in order design the experience of users.

\subsubsection{Quality Function Deployment}

QFD was developed by Akao in Japan in 1966 and is defined as a method for developing a design quality aimed at satisfying the consumer and then translating the consumers' demand into design targets and major quality assurance points to be used throughout the production phase [7]. QFD is a cross-functional planning tool that can be used to deploy the voice of the customer from product planning, designing, engineering and manufacturing into a final product. The Quality Function Deployment interaction matrix was used to effectively define the customer requirements and convert them into detailed specifications and plans to produce the prototype. It helped to understand the needs of the users of the multi-sided platform and understand the engineering capabilities that were going to be needed to develop the prototype. Fig. 4 shows the final matrix obtained through the analysis. 


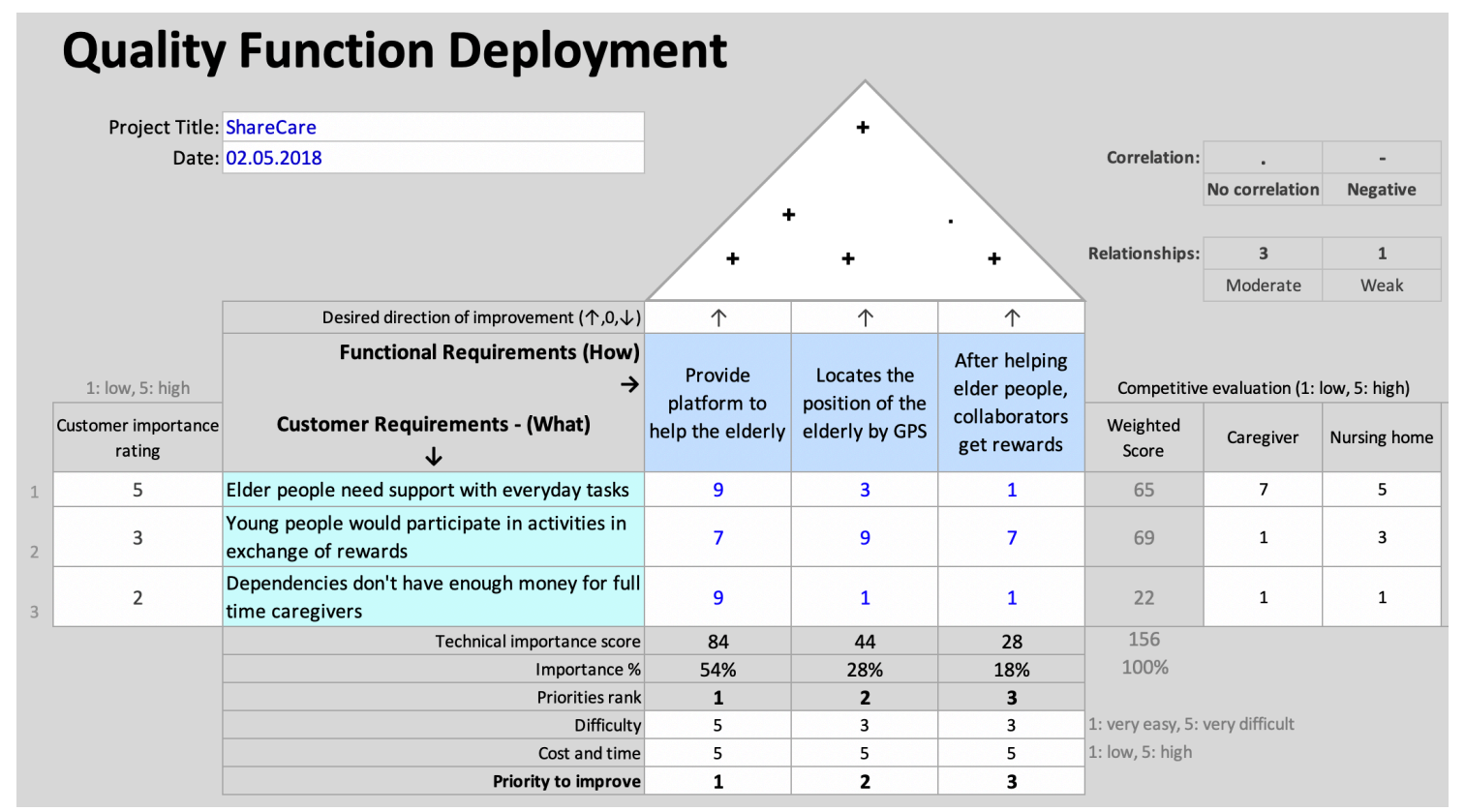

Fig. 4. Quality Function Deployment Matrix for ShareCare

\subsubsection{Persona Analysis}

Creating personas helps to understand the users' needs, experiences, behaviors and goals. It's also a practice during an innovation process for analyzing the service and its components since it helps to create a good user experience to the targeted user group [8]. A persona analysis was done for this research to examine the process and workflow that the user would prefer to use in order to achieve their objectives in interacting with this service. This were the different personas of the target user drawn from the analysis:

- Persona 1 (Mary): Mary is a granny. She is in her 80s. As she's getting older and weaker everyday tasks are harder to do. She lives in Taiwan and her family recently moved to the US. She would appreciate a little help, but he just doesn't know who to ask for it.

- Persona 2 (Tim): Tim is a freshman student from a master's degree program. He is a kindhearted young man and he likes to help people. As a student, his resources are limited and so he is always in the lookout of coupons for purchasing food and clothe.

- Persona 3 (Mr. Lee): Mr. Lee is CEO of a starting bubble tea company. He wants to create his company a good name and participate in activities that support his community. $\mathrm{He}$ knows that by offering some discounts, his firm will show up at the market, be recognized as socially responsible and sell much more bubble teas!

\subsubsection{Customer Journey Map}

A CJM (Customer Journey Map) is a widely used tool to represent user experience with a service [9]. Fig. 5 shows the customer journey map that was drawn in this research to highlight the touch points that influence in the different decision-making processes to understand and visualize the customer's experience. The CJM provided insights that were helpful to determine the touch points in which we had to pay more attention and focus, like the Human-Computer interaction between the service design and the users. 


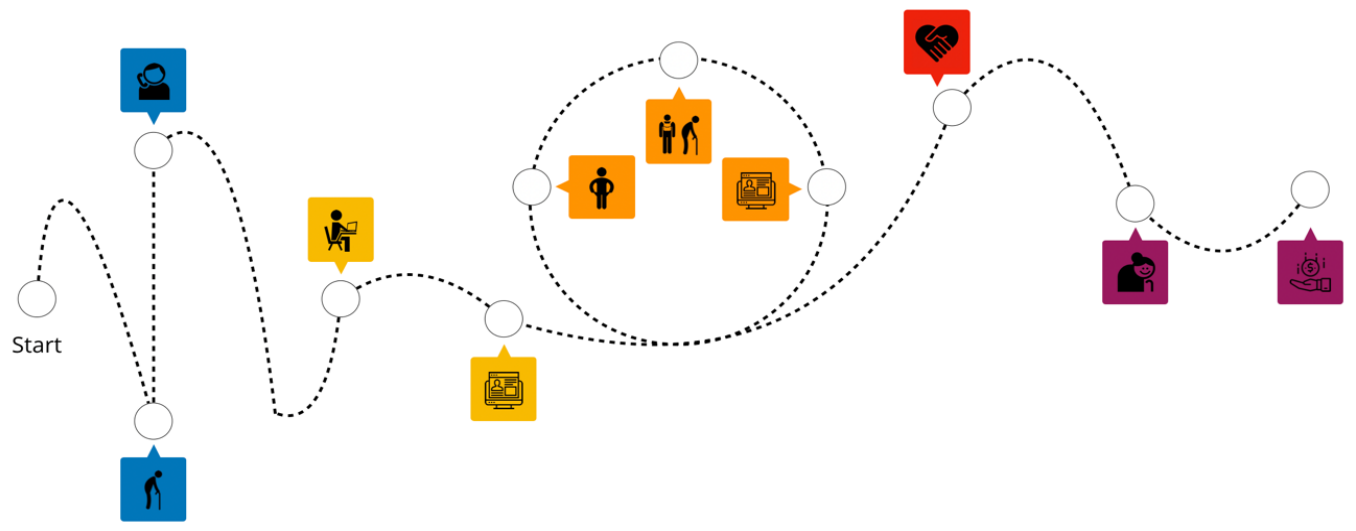

Fig. 5. Customer Journey Map for ShareCare

\subsubsection{Service Blueprint}

Service blueprinting upholds the focus of a service innovation on the human-to-human and human-to-technology interfaces at the project boundaries, rather than at the software engine level, allowing service designers to drill down into the project without losing the connection to customer actions and process [10]. A service blueprint was developed to understand the service in terms of how it will be provided, specifying the physical evidence, staff actions, and support systems / infrastructure needed to execute this project across its different channels. Fig. 6 shows the drawn blueprint. Through the obtained information we were able to identify that the process of registration required more importance according to the users that we were designing for since it's a project designed for elderly who encounter barriers using technology sometimes.

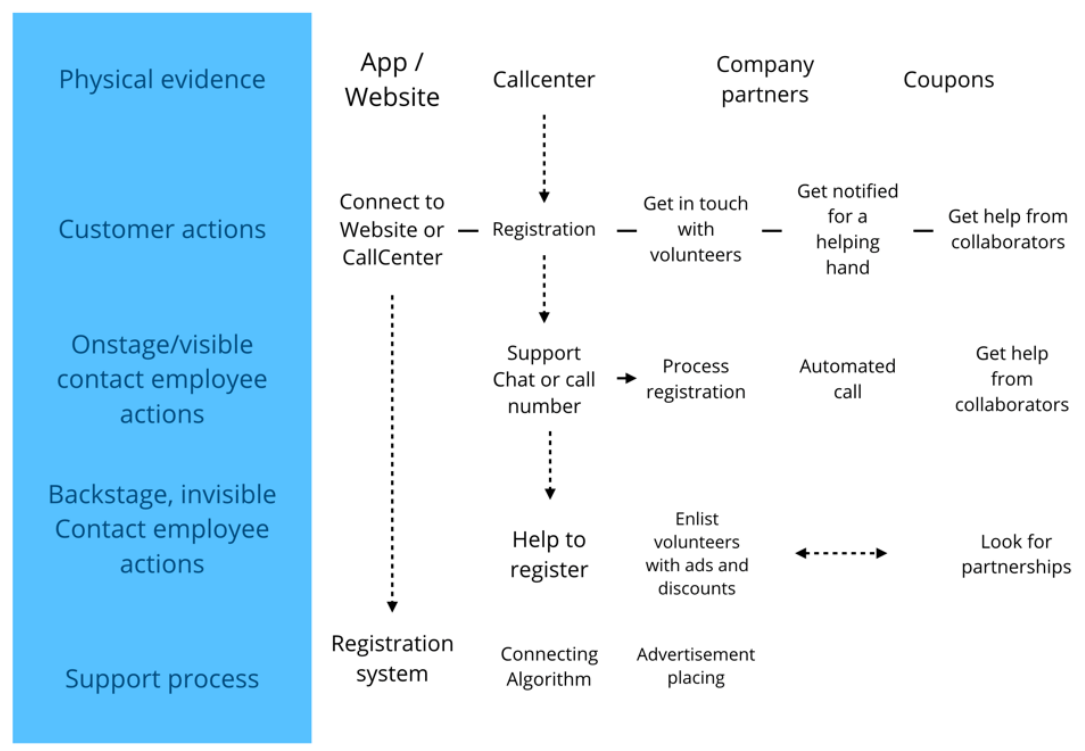

Fig. 6. Service Design Blueprint for ShareCare 


\subsection{Evaluation}

\subsubsection{Analytic Hierarchy Process}

The AHP allows decision makers to model complex problems in a hierarchical structure depending on the relationship among the main objective of the problem, criteria, sub- criteria and scenarios [11].

As a way to elaborate an evaluation plan for the execution and the development of the platform an Analytic Hierarchy Process model was drawn. This was helpful since it served as a multi-criteria decision-making method for organizing the efforts and decisions needed. It helped to understand the necessary focus in the app and platform development based on factors like easy to use interaction and cost. Fig. 7 shows the exercise of the drawn process.

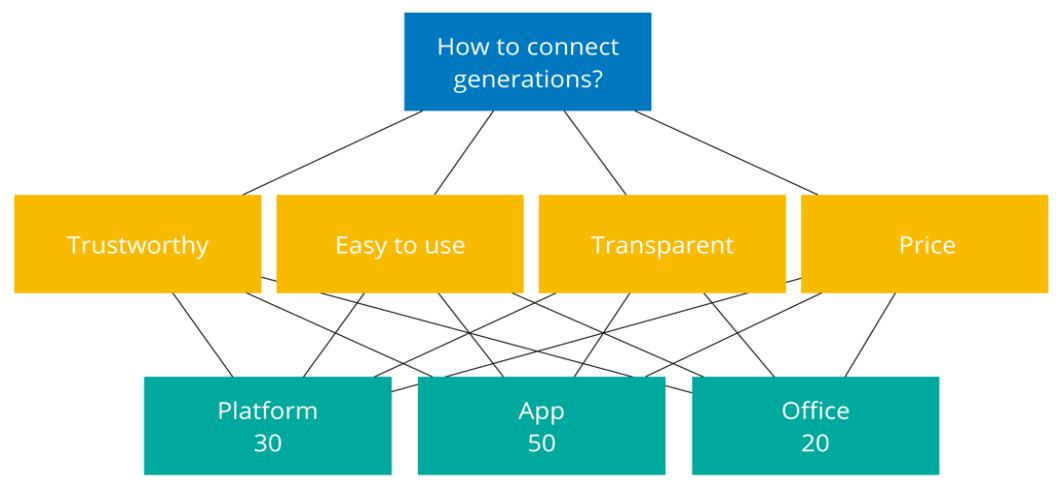

Fig. 7. Analytic Hierarchy Process for ShareCare

\subsubsection{Kano Model Analysis}

The Kano model screens important service function attributes and studies the nature of customer needs by providing a way for a better classification of customer needs [12]. A Kano model analysis was also done to help determine which features to include in the service to improve the customer satisfaction. Fig. 8 shows how technology and functionality were aspects to be highly considered in the user experience design process and how this should be prioritized to guarantee the efficiency and affordance of the service according to the results obtained from the model.

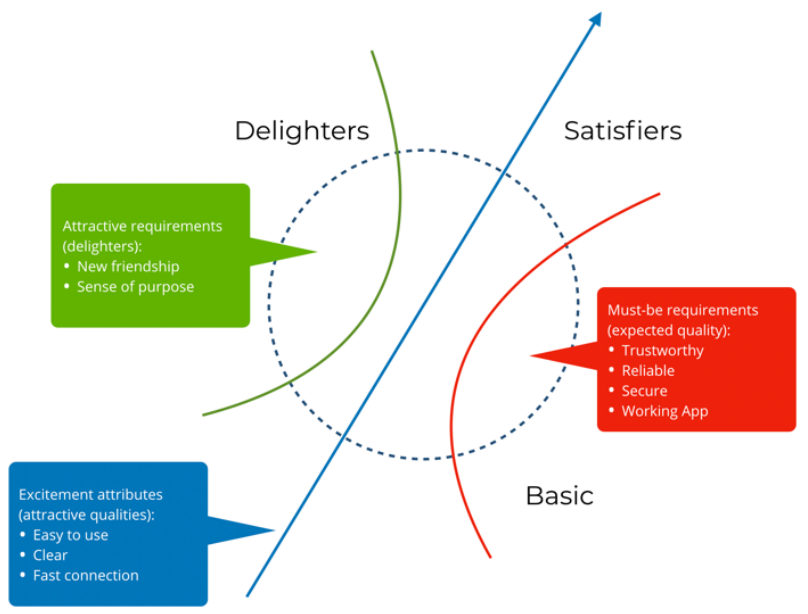

Fig. 8. Kano Model Analysis for ShareCare 


\subsubsection{Business Model Canvas}

The BMC (Business Model Canvas) by Strategyzer describes the rationale of how an organization creates, delivers and capture value [13]. It provides a common framework for organizations to understand the different purposes of the many information sources available and proposes an iterative process of making and testing assumptions against research [14].

The BMC was helpful to understand the relationships and activities required to validate the innovation of the service. It was also useful to understand the resources and tasks needed to execute the project. This analysis helped develop a service logic-oriented framework for innovative business model development. Fig. 9 shows the results of the traced canvas based on the project Share Care.

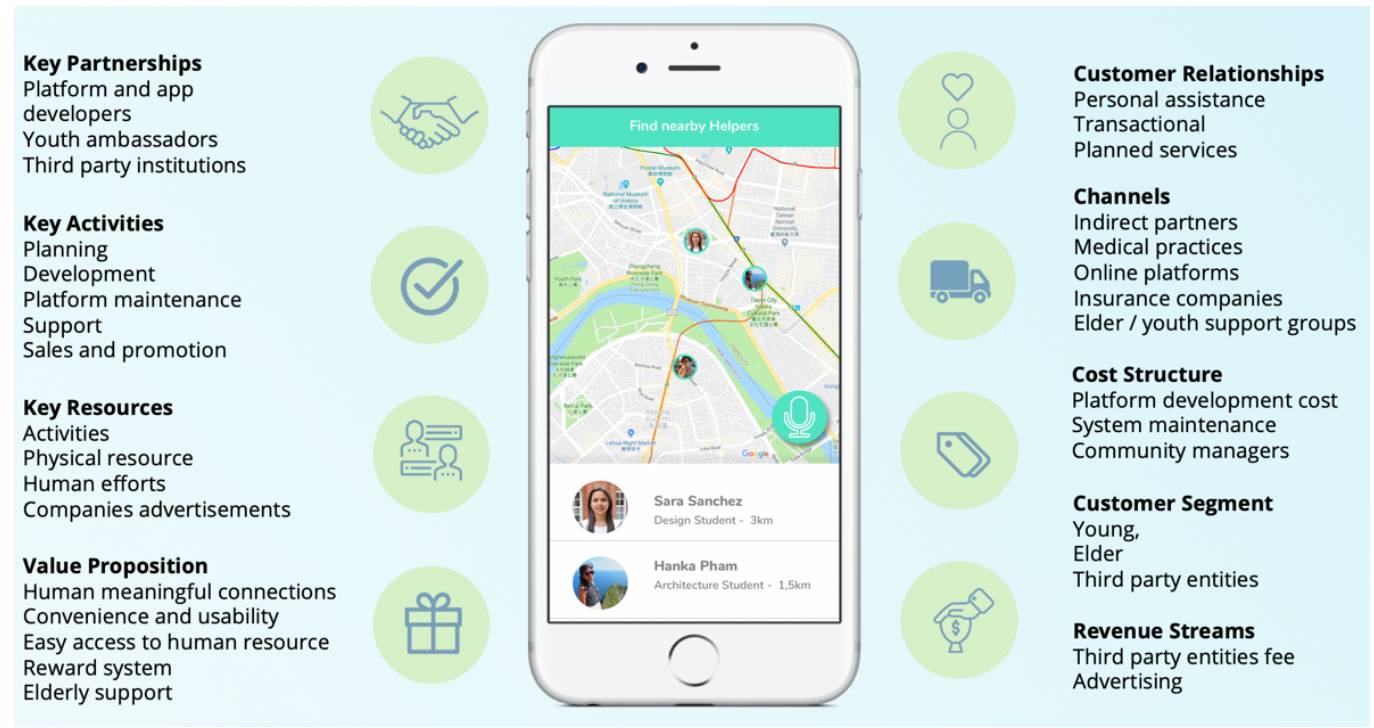

Fig. 9. Business Model Canvas for ShareCare

4. System Demonstration and Research Results

\subsection{Technology Specification and Research Results}

The time-sharing platform proposes a solution based on the identified difficulties encountered by the elderly in their daily lives and proposes a system that integrates youngsters' support. ShareCare is a mobile app that provides real-time support to seniors by locating young helpers that want to help them exchange of rewards. Seniors can search for nearby helpers and helpers can also use this function to find the elderly within distance to provide assistance.

\subsection{Prototype and User Interface Demonstration}

Sketch and Flinto were used to create a high-fidelity prototype to show the operation process of ShareCare. The first interface was used to validate the operation flow and make corrections according to the feedback that were given by the user. Google Voice Assistant services were incorporated to interact with the senior users and support them along onboarding process. Fig. 10 and 11 show the interfaces designed for elder adults and youngsters respectively. 


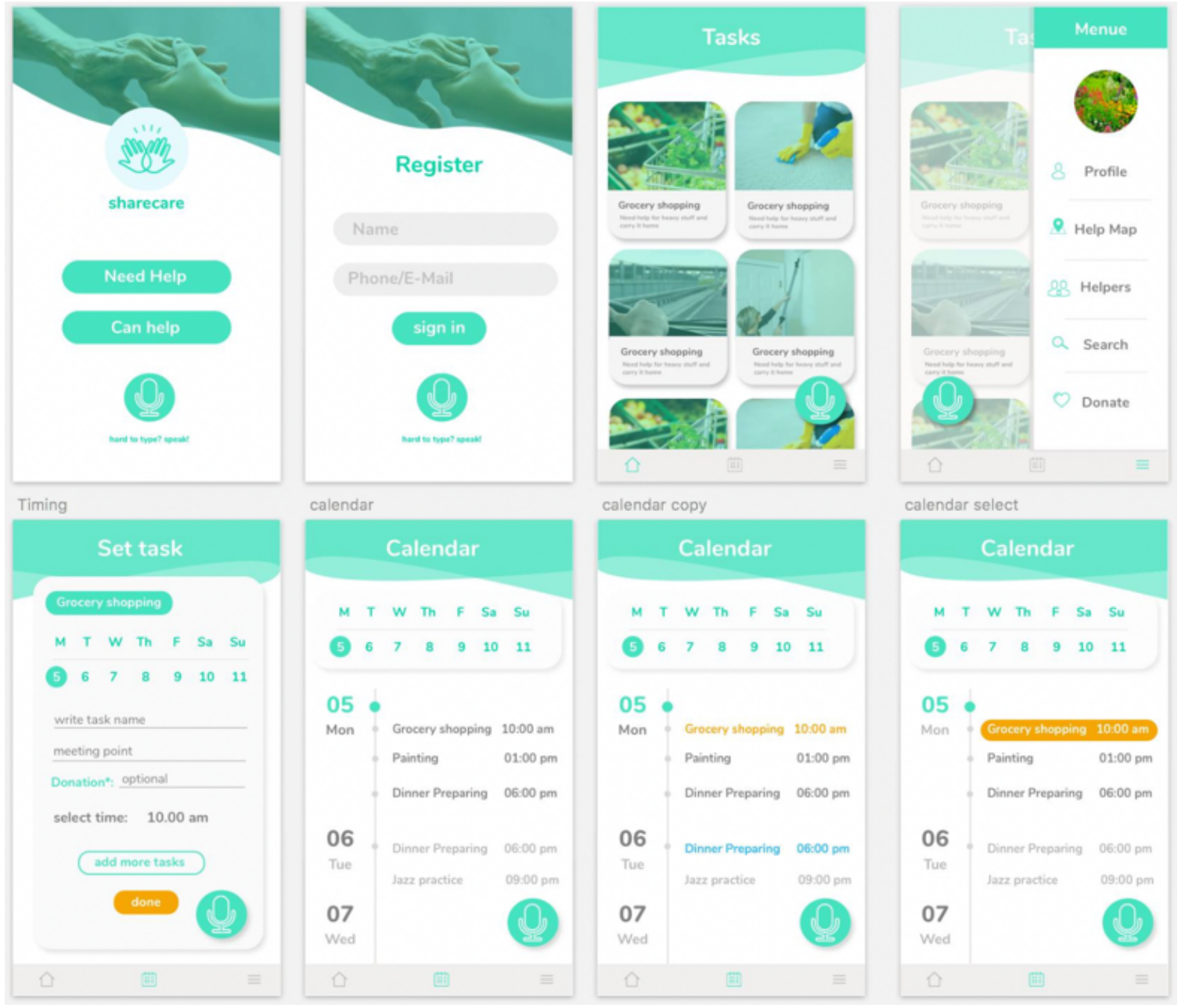

Fig. 10. ShareCare mobile app interface for elderly

\subsection{User Interface and Innovation Evaluation}

Peter Morville's user experience honeycomb is a tool that explains the various facets of user experience design. It helps to find "a sweet spot" between the various areas of a good user experience [15]. The honeycomb theory was used to validate the value, utility, and affordance of the system as illustrated in Tab. 1.

\begin{tabular}{|l|l|}
\hline Theory & Prototype \\
\hline Useful & $\begin{array}{l}\text { ShareCare addresses the clear need that elderly need and the app creates a } \\
\text { user-friendly platform that brings a better experience for the target market. }\end{array}$ \\
\hline Useable & $\begin{array}{l}\text { The service is a straightforward and easy to use platform that does not } \\
\text { incorporate extra features that might be confusing. It's clean and direct. } \\
\text { Incorporates familiar patters for login in to the app. }\end{array}$ \\
\hline Findable & $\begin{array}{l}\text { App is easy to navigate, and information is accessible for the different users. } \\
\text { Platform is an easy-to-navigate app for elder and youngsters that does not } \\
\text { require time consuming research. }\end{array}$ \\
\hline Credible & $\begin{array}{l}\text { ShareCare builds trust by monitoring user's feedback from both sides, young } \\
\text { and elder which builds confidence and helps other make the decision to } \\
\text { continue using the product. }\end{array}$ \\
\hline Accessible & $\begin{array}{l}\text { No matter their age or disabilities, ShareCare is designed to understand the } \\
\text { habits of its user and make the interaction possible for everyone. }\end{array}$ \\
\hline Desirable & $\begin{array}{l}\text { The visual interface and appearance are attractive, simple and easy to } \\
\text { understand. They help the elder and the younger to achieve their goal. The } \\
\text { service is well presented and inviting. }\end{array}$ \\
\hline
\end{tabular}

Tab. 1. Honeycomb analysis for ShareCare 


\section{Conclusion}

\subsection{Briefing of Case Study}

The elderly population is gradually rising. The percentage of elderly people in Taiwan, Asia and the world continues to increase through the years and innovative management systems need to be created to support them. As the elderly population grows older, their physical functions are easily degraded. In daily life, they will encounter many difficulties, such as the loss of strength in the hands and the inability to extract heavy objects. Some will even have difficulties in going out and complete some of their everyday tasks. Young people are often looking for opportunities to do a better usage of their time and resources.

Based on an interdisciplinary service design process we present an innovative proposal and a prototype for intergenerational time-sharing management. ShareCare's serves as an intergenerational platform that enabled by technology connects older adults and youngsters through task management and collaboration.

\subsection{Conclusion}

Based on the research and analysis, ShareCare is our proposal of an innovative time-sharing management service that supports the elderly with the difficulties encountered in daily tasks. It's also serves as a platform for youngsters to support elder adult and be rewarded for their support. Under the trend of an aging society and technology services our system's contribution is focused in the aspect of doing social innovation design for providing a platform to connect people for intergenerational interaction and communication.

In the long run, a system like this can help a society to promote exchanges between seniors and young people, reduce the distance between each other, and make a more harmonious community.

Combining the above service design analysis and reports with the qualitative feedback from potential users, ShareCare is a service that proves to be helpful for elder people to have a more convenient and supported life. With the support of "Young helpers", the platform can reduce the difficulties experienced by seniors in their daily lives. Seniors can quickly get support and assistance in the daily tasks they need help with and in order to promote a mutual support channel, ShareCare can also reward with points every time young helpers complete their tasks as well. Volunteers can therefor exchange reward points for coupons and discounts with ShareCare partners like restaurants and clothing stores.

\subsection{Suggestion for future works}

A qualitative research was done for this project and it proved to be a useful methodology and tool for system's innovation management. The analysis done shows that design service analysis tools are helpful in the design of two user interfaces that were clearly design and studied to fit the purpose of the project. Through this conceptual analysis, a more quantitative method could be applied to collect data that validates the user experience efficiency and the complexity of the system. Also, for future works fast iteration and implementation could be introduced in a greater way to understand the processes and possible scalability of the project. 


\section{References}

[1] Jansen TR. When Preschool Is in a Nursing Home. 2016 January. Retrieved September 18, 2018 from https://www.theatlantic.com/education/archive/2016/01/the-preschool- inside-a-nursinghome $/ 424827 /$.

[2] Chao TY, Yang* YY. Ageing in Place: Successful Housing Community for Elderly in Taiwan - A Case Study of Taipei City. 2011. Available from: 10.5353/th_b5372741.

[3] Collman A. Daycare within a nursing home where youngsters sing, color and read with the elderly. 2015 October. Retrieved September 10, 2018 from https:/www.dailymail.co.uk/news/article3285008/Pensioner-playmates-amazing- daycare-nursing-home-youngsters-sing-color-readelderly.html.

[4] MyBestHelper. (n.d.). Retrieved September 10, 2018, from https://www.mybesthelper.com/

[5] Dam, R., \& Siang, T. (n.d.). 5 Stages in the Design Thinking Process. Retrieved September 18, 2018 from https://www.interaction-design.org/literature/article/5-stages-in-the-design-thinking-process

[6] What is Service Design?| Designlab blog. (n.d.). Retrieved November 10, 2018, from https://trydesignlab.com/blog/what-is-service-design/

[7] Becker Associates Inc. Retrieved September 10, 2018 from http://www.beckerassociates.com/thehouse.HTM and http://www.becker-associates.com/qfdwhatis.htm

[8] Dam, R., \& Siang, T. (n.d.). Personas - A Simple Introduction. Retrieved November 08, 2018 from https://www.interaction-design.org/literature/article/personas-why-and-how-you-should-use-them

[9] H., Han, S. H., Chun, J., \& Hong, S. W. (2016). A Design Process for a Customer Journey Map: A Case Study on Mobile Services. Human Factors and Ergonomics in Manufacturing \& Service Industries,26(4), 501-514. doi:10.1002/hfm.20673

[10] Bitner, M. J., Ostrom, A. L., \& Morgan, F. N. (2008). Service Blueprinting: A Practical Technique for Service Innovation. California Management Review,50(3), 66-94. doi:10.2307/41166446

[11] Orman A, Düzkaya H, Ulvi H, et al. Academic Search Complete. Gazi University Journal of Science. 2018;31(2):381-397.

[12] Ji P, Jin J, Wang T, et al. International Journal of Production Research. International Journal of Production Research. 2014;52(21):6335-6348. Base de datos: Business Source Complete; Available from: 10.1080/00207543.2014.939777

[13] Osterwalder Y. Business Model Generation. New Jersey: John Wily \& Sons. Inc; 2010.

[14] Oneill TW. The business model canvas as a platform for business information literacy instruction. Reference Services Review. 2015;43(3):450-460. Available from: 10.1108/rsr-02-2015-0013.

[15] Wesolko, Dane. "Peter Morville's User Experience Honeycomb - Dane Wesolko Medium.” Medium, Medium, 14 June 2016, medium.com/@danewesolko/peter-morvilles-userexperience-honeycomb-904c383b6886. 